\title{
The Use of Small and Wide Angle X-Ray Scattering (SWAXS) for the Physico-Chemical Characterization of Solid Dispersions
}

\author{
M. MetZler ${ }^{1}$, A. Hodzic ${ }^{1}$, P. LAggneR ${ }^{2,3}$, N. A. URbanetz ${ }^{1,4}$ \\ ${ }^{1}$ Research Center Pharmaceutical Engineering GmbH, Graz, Austria \\ ${ }^{2}$ Institut of Biophysics and X-Ray Structure Research, Austrian Academy of Sciences, Graz, Austria \\ ${ }^{3}$ HECUS X-Ray Systems, Graz, Austria \\ ${ }^{4}$ Institute for Process and Particle Engineering, Graz University of technology, Graz, Austria \\ E-mail: aden.hodzic@tugraz.at (A. Hodzic)
}

Sci Pharm. 2010; 78: 691

doi:10.3797/scipharm.cespt.8.PPAT02

\begin{abstract}
Solid dispersions of active pharmaceutical ingredients (APIs) in freely water soluble carriers are often used in order to improve dissolution rate and/or solubility of poorly water soluble drugs [1] in the aqueous gastro intestinal fluids. Depending on the dispersion of the drug in the carrier one may distinguish between solid solutions, in which the drug is molecularly dispersed within the carrier and solid suspensions, which is a dispersion of either amorphous or crystalline drug particles in the carrier. Whether a solid solution or a solid suspension is obtained, depends on the preparation conditions of the solid dispersion. Moreover, storage may lead to changes in the dispersion of the drug in the carrier, for example leading to the recrystallisation of initially dissolved API in the carrier. These changes may substantially alter the dissolution profile/solubility of the drug in the aqueous dissolution media.

There are several techniques that are used in order to either determine the state of dispersion of the drug in the carrier or to monitor storage induced changes. Conventional techniques include thermal analysis and wide angle $X$ ray diffraction, although the detection of very fine drug crystals in the carrier or the transition of an initial solid solution to a solid suspension at a very early stage is almost impossible.

We apply simultaneous small and wide-angle X-ray scattering, which is becoming more important for solid-state pharmaceutical analytics [2], in order to gain information of solid nano-structure and dispersions stability. The study presents examples consisting of nimodipine and polyethylene glycol and/or polyvinylpyrrolidone, characterized by SWAXS analytical parameters.
\end{abstract}

[1] Chiou WL, Riegelmann S. Pharmaceutical Applications of Solid Dispersion Systems. J Pharm Sci. 1971; 60: 1505-1510. doi:10.1002/jps.2600600902

[2] Hodzic A, Kriechbaum M, Laggner P. Laboratory SWAXS for application in the pharmaceutical technology. Acta Cryst A. 2009; A65: S120. 